\title{
Improving the performance of optical burst switching with large control overhead
}

\author{
C. Y. Li, ${ }^{1}$ P. K. A. Wai, ${ }^{1}$ and Victor O. K. $\mathrm{Li}^{2}$ \\ ${ }^{1}$ Department of Electronic and Information Engineering, The Hong Kong Polytechnic University, \\ Hong Kong, China, \{enli, enwai\}@polyu.edu.hk \\ ${ }^{2}$ Department of Electrical and Electronic Engineering, The University of Hong Kong, \\ Hong Kong, China, vli@eee.hku.hk
}

\begin{abstract}
In optical burst switching (OBS) system, the throughput decreases rapidly with increase in control packet processing time $T_{\mathrm{cp}}$. The negative impact of $T_{\mathrm{cp}}$ will become significant as the optical fiber transmission rate increases. By analyzing the relationship between the throughput and $T_{\mathrm{cp}}$, we attempt to improve the throughput. Different possible solutions are discussed. We found that using extra random offset time can significantly improve the throughput at the expense of increase in data burst delay.
\end{abstract}

Index Terms-optical burst switching, optical packet switching, control overhead, offset time

\section{INTRODUCTION}

Without practical optical signal processing devices and optical buffers, all-optical packet switching is still in the early research stage [1]. Recently, optical burst switching (OBS) becomes popular because it can provide connectionless type of transmission services with low optical hardware complexity [2-6]. The traffic between OBS nodes are data bursts, each consisting of multiple packets. One-way reservation is used for the data transmission in OBS networks. When a data burst is generated at a node, a control packet is sent immediately to the destination of the data burst. The control packet reserves the resources at the nodes on the path of the data burst. No acknowledgment is sent back in order to minimize the delay time of sending out the data burst at the source. After an offset time, the node sends out the data burst following the same routing path of the control packet. If the reservation by the control packet is successful, the data burst will pass through all nodes on the path without any processing and optical-to-electrical $(\mathrm{O} / \mathrm{E})$ conversion. No optical buffers are required in the intermediate nodes, thus significantly simplifying the implementation of optical routing nodes.

One-way reservation is used in OBS networks as it is assumed that the average data burst transmission time can be much shorter than the propagation delay between nodes [2]. Also, the processing time $T_{\mathrm{cp}}$ of the control packets at intermediate nodes can be significant when compared to the transmission time of the data bursts. In these situations, feedback acknowledgment (two-way reservation) approach will be very

This research is supported by a grant from The Hong Kong Polytechnic University (Proiect Number 1-BBZB). inefficient. Using one-way reservation, the large propagation delay between nodes will no longer be a major concern for system performance. OBS networks can therefore perform better than the connection-oriented wavelength-routed optical networks.

Since the offset time $T_{\text {off }}$ between the transmission of a data burst and its control packet must be larger than the total processing time of the control packet on the path, simple OBS channel reservation schemes such as Horizon [3] and just-in-time (JIT) [4] become inefficient in transmission bandwidth utilization when $T_{\mathrm{cp}}$ increases. Although sophisticated channel reservation schemes such as the last available unused channel with void filling (LAUC-VF) [5] scheme are more bandwidth efficient, and in principle can reuse all the idle transmission capacity in the time interval of $T_{\text {off, }}$ the system throughput will still degrade significantly if $T_{\mathrm{cp}}$ is large. To eliminate the negative impact of control overhead, one may delay the data bursts at the inputs of intermediate nodes to compensate the control packet processing time but fiber delay lines (FDLs) are required [2], [3].

The control packet processing time $T_{\mathrm{cp}}$ will become increasingly significant because the data burst transmission time will decrease with the increase in the transmission rate of optical fiber, and one cannot lengthen the data bursts by too much. When trying to improve the system performance, it is important to preserve the low optical hardware complexity characteristics of OBS. In addition, a thorough understanding of the relationship between $T_{\mathrm{cp}}$ and the OBS performance is required. So far, control overhead is assumed to be small in OBS networks and is often neglected in the derivation of OBS schemes and performance evaluation. When $T_{\mathrm{cp}}$ is not set to zero, however, the focus is almost always on the whole offset time $T_{\text {off }}$ and little attention has been given to the role of $T_{\mathrm{cp}}$ in the system performance.

In this paper, we study the relationship between $T_{\mathrm{cp}}$ and OBS performance and investigate means to improve OBS performance at large $T_{\mathrm{cp}}$. In Section II, we review the degradation of OBS network performance when the control overhead increases. We observe that the system throughput degrades rapidly when the control packet processing time $T_{\mathrm{cp}}$ increases but it becomes insensitive to the increase of $T_{\mathrm{cp}}$ when $T_{\mathrm{cp}}$ is larger than the data burst transmission time $L$. In Section III, we study the 
role of $T_{\mathrm{cp}}$ in the throughput performance degradation. We then discuss methods that improve the throughput performance in Section III. In Section IV, we show that the throughput can be significantly increased by using the random extra offset but the data burst delay will also increase. We conclude in Section V.

\section{REVIEW OF OBS Throughrut PERFormance}

Figure 1 shows the throughput performance of OBS in an 8 $\times 8$ Manhattan Street Network (MSN) [7] with LAUC-VF channel reservation scheme and different control overhead. The OBS with Horizon [3] and JIT [4] channel reservation schemes are not included because these two schemes do not reuse the transmission bandwidth in the time interval of $T_{\text {off }}$ between the data burst and control packet. It is therefore not surprising that their throughput performance decreases when $T_{\mathrm{cp}}$ increases, i.e., $T_{\text {off }}$ increases. In contrast, LAUC-VF channel reservation scheme in principle can reuse all the idle transmission bandwidth. Intuitively, LAUC-VF should not be sensitive to $T_{\mathrm{cp}}$. However, simulations often show that LAUC-VF throughput performance severely degrades if $T_{\mathrm{cp}}$ is large, especially in networks having paths with large number of hops.

Solid lines with crosses, circles, and squares in Fig. 1 represent the system throughputs when the control packet processing times are $0.1,1.0$ and 10 times of the data burst transmission time. For comparison, the dashed line corresponds to $T_{\text {cp }}=0$. In the simulations, the offset time between a data burst and its control packet is given by

$T_{\text {off }}=H \times T_{\text {cp }}+T_{\text {sw }}$,

where $H$ is the number of hops to the destination from the current location of the control packet [2]. Hence, $H$ is equal to the total hop count of the path when the control packet is at the source, and decreases by one every time the control packet passes an intermediate node. The time $T_{\mathrm{sw}}$ is the required switch reconfiguration time at each node. Equation (1) assumes that a control packet leaves a node immediately after the node finds a channel for the corresponding data burst. There is no need to wait for the switch to reconfigure. Without special node architecture and switch reconfiguration algorithms, $T_{\mathrm{sw}}$ can also cause large throughput loss [6]. In this paper, we will focus on the impact of $T_{\mathrm{cp}}$ and set $T_{\mathrm{sw}}=0$ in all simulations. For ease of discussion, we normalized $T_{\mathrm{cp}}$ by the data burst transmission time in the following discussion. Other assumptions of the simulations are given in Section IV.

In Fig. 1, the OBS LAUC-VF with $T_{\mathrm{cp}}=0.0$ have a maximum throughput of 0.22 at a loading of 0.4 and reduces to 0.2 when the loading approaches one. On the other hand, OBS LAUC-VF with $T_{\mathrm{cp}}=0.1$ and 1.0 have the maximum throughputs of 0.18 and 0.16 , respectively, at loadings of 0.3 and 0.2 , and have a throughputs of 0.05 and 0.01 , respectively, when the loading approaches one. Obviously, large $T_{\mathrm{cp}}$ has a severe negative impact on the OBS system throughput performance. However, when $T_{\mathrm{cp}}$ increases beyond 1.0, the throughput performance of

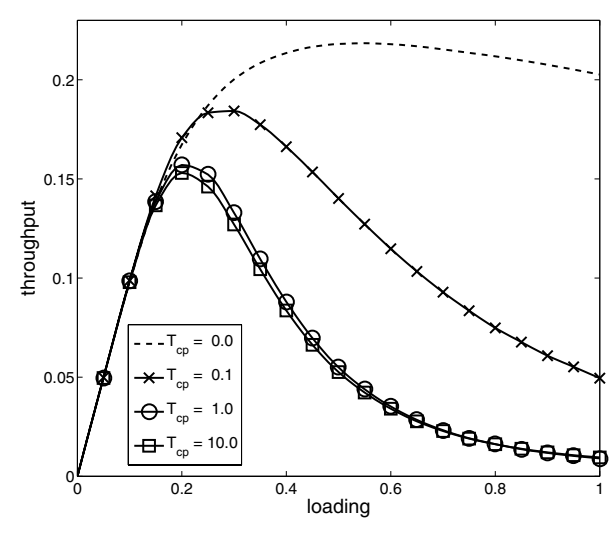

Fig. 1 The OBS throughput performance of an $8 \times 8$ Manhattan Street Network (MSN) with LAUC-VF for different control overheads. Solid lines with crosses, circles, and squares are the system throughputs when the control packet processing times are $0.1,1.0$ and 10 times of the data burst transmission time respectively. The dashed line represents the throughput for $T_{\mathrm{cp}}=0$. The setting and assumptions of the simulations are described in Section IV.

LAUC-VF is similar to that of $T_{\mathrm{cp}}=1.0$ even if $T_{\mathrm{cp}}>>1$, e.g., $T_{\mathrm{cp}}$ $=10.0$ in Fig. 1. The throughput of LAUC-VF appears to be insensitive to the value of $T_{\mathrm{cp}}$ when $T_{\mathrm{cp}}$ is larger than the data burst transmission time.

It seems that the results observed in Fig. 1 are due to the priority effect of the offset time $T_{\text {off }}$ [8]. Assuming random control packet arrival, a data burst is more likely to be blocked by a data burst with a large $T_{\text {off }}$ than by one with a small $T_{\text {off. According to }}$ Eq. (1), data bursts with larger hop count paths will find it easier to reserve the output channels at nodes in the early stage of their journey, but they are also likely to be blocked when they are close to their destinations. Much transmission bandwidth is therefore wasted. A larger $T_{\mathrm{cp}}$ seems to enhance this offset time priority effect. Although this explanation seems valid for the throughput curves of $T_{\mathrm{cp}}=0.0,0.1$ and 1.0, it is not sufficient to explain the curve of $T_{\mathrm{cp}}=10.0$ shown in Fig. 1. A more fundamental OBS performance model based on the control packet processing time $T_{\mathrm{cp}}$ is required.

\section{THE MODEL}

Figure 2 shows two control packets $\mathrm{CP}_{1}$ and $\mathrm{CP}_{2}$ and their corresponding data bursts $\mathrm{DB}_{1}$ and $\mathrm{DB}_{2}$. We assume that the two control packets arrive at the input ports of a node $N_{\mathrm{j}}$ and request the same output port $O_{\mathrm{k}}$. If there are multiple channels available at the output port, LAUC-VF chooses the idle time gap in the channels that can accommodate and has the start time closest to the arrival of the data burst [5]. For simplicity, we assume that there is only one wavelength channel per output port and all data bursts have constant transmission time $L$. The discussion can be easily generalized to multiple channel OBS with variable burst length. In Fig. 2, we assume that the arrival time of the control packets $\mathrm{CP}_{1}$ and $\mathrm{CP}_{2}$ are $t_{1}$ and $t_{2}$, respectively, where $t_{1} \leq t_{2}$. The offset times associated with the control 


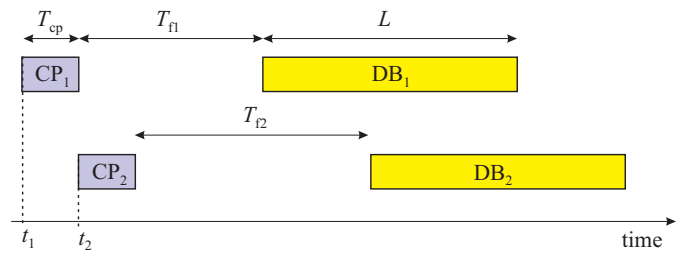

Fig. 2 Two control packets $\mathrm{CP}_{1}$ and $\mathrm{CP}_{2}$ and their corresponding data. bursts $\mathrm{DB}_{1}$ and $\mathrm{DB}_{2}$.

packets are $T_{\mathrm{fl}}$ and $T_{\mathrm{f} 2}$ respectively. We assume a first-come-first-serve (FCFS) discipline. Since the control packet $\mathrm{CP}_{1}$ arrives at the node earlier, $\mathrm{CP}_{1}$ will block the channel request of $\mathrm{CP}_{2}$ if $t_{1}, t_{2}, T_{\mathrm{fl}}, T_{\mathrm{f} 2}$, and $L$ satisfy

$t_{2}+T_{\mathrm{f} 2}-L<t_{1}+T_{\mathrm{fl}}<t_{2}+T_{\mathrm{f} 2}+L$.

Eq. (2) simply gives the condition that part of $\mathrm{DB}_{1}$, either its head or tail, will overlap with $\mathrm{DB}_{2}$ if the request of $\mathrm{CP}_{1}$ is accepted. We consider the two cases (i) $T_{\mathrm{f} 1} \leq T_{\mathrm{f} 2}$, and (ii) $T_{\mathrm{fl}}>T_{\mathrm{f} 2}$ separately. In case (i), we can simplify Eq. (2) to

$t_{2}+\left(T_{\mathrm{f} 2}-T_{\mathrm{f} 1}\right)-L<t_{1} \leq t_{2}$,

because $t_{2}+\left(T_{\mathrm{f} 2}-T_{\mathrm{f} 1}\right)+L$ is larger than $t_{2}$ and $t_{2} \geq t_{1}$. Since $\mathrm{CP}_{2}$ $\left(\mathrm{DB}_{2}\right)$ will not be blocked if $T_{\mathrm{f} 2}>\left(T_{\mathrm{f} 1}+L\right)$, Eq. (3) explains the mechanism of OBS systems that support priority traffic classes with extra offset time: a high priority data burst will not be blocked by any low priority data burst if its offset time is larger than the sum of the data burst length and the maximum offset time of the low priority traffic. In case (ii), i.e., $T_{\mathrm{f} 1}>T_{\mathrm{f} 2}$, Eq. (2) can be rewritten as

$t_{2}-\left(T_{\mathrm{fl} 1}-T_{\mathrm{f} 2}\right)-L<t_{1}<\min \left[t_{2}, t_{2}-\left(T_{\mathrm{fl}}-T_{\mathrm{f} 2}\right)+L\right]$.

From Eqs. (3) and (4), the length of the intervals in which $\mathrm{CP}_{1}$ will block $\mathrm{CP}_{2}\left(\mathrm{DB}_{2}\right)$ are given as $\max \left[\left(L-\left|T_{\mathrm{fl}}-T_{\mathrm{f} 2}\right|\right), 0\right]$ and $\min \left[\left(L+\left|T_{\mathrm{f} 1}-T_{\mathrm{f} 2}\right|\right), 2 L\right]$, respectively. Please note that the time interval in which blocking will occur for case (ii) $\left(T_{\mathrm{fl}}>T_{\mathrm{f} 2}\right)$ is always larger than that of case (i) $\left(T_{\mathrm{f} 1} \leq T_{\mathrm{f} 2}\right)$. Also, adding a constant extra offset time to all data bursts will not affect the system throughput because the constant extra offset time does not change the results in Eqs. (3) and (4).

In order to study the role of $T_{\mathrm{cp}}$ in the throughput performance of OBS, we substitute Eq. (1) into Eqs. (3) and (4). The interval within which $t_{1}$ occurs such that $\mathrm{CP}_{1}$ will block $\mathrm{CP}_{2}\left(\mathrm{DB}_{2}\right)$ becomes $\max \left[\left(L-\left|H_{1}-H_{2}\right| \times T_{\mathrm{cp}}\right), 0\right]$ for $H_{1} \leq H_{2}$ and $\min \left[\left(L+\mid H_{1}\right.\right.$ $\left.\left.-H_{2} \mid \times T_{\mathrm{cp}}\right), 2 L\right]$ for $H_{1}>H_{2}$. Figure 3 plots the length of the interval within which $t_{1}$ occurs such that $\mathrm{CP}_{1}$ will block $\mathrm{CP}_{2}$ $\left(\mathrm{DB}_{2}\right)$ for different $\left(H_{2}-H_{1}\right)$ and $T_{\mathrm{cp}}$. The blocking time interval and $T_{\mathrm{cp}}$ in Fig. 3 are normalized by the data burst transmission time $L$. The lines with pluses and asterisks are the time interval length of $T_{\mathrm{cp}}=0.1 L$ and $0.2 L$, respectively. The line with circles is that of $T_{\mathrm{cp}} \geq L$. From Fig. 3, the blocking time interval is $L$ when $H_{2}=H_{1}$. For $H_{2}>H_{1}$ the time interval decreases linearly and becomes zero when $\left(H_{2}-H_{1}\right) \geq L / T_{\text {cp }}$. As $T_{\text {cp }}$ increases, the range of $\left(H_{2}-H_{1}\right)$ for non-zero blocking time interval decreases

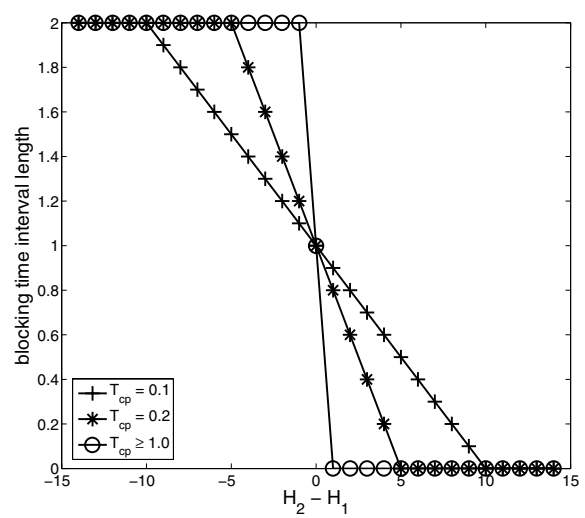

Fig. 3 The length of the blocking time interval for $\mathrm{CP}_{1}$ to block $\mathrm{CP}_{2}$ $\left(\mathrm{DB}_{2}\right)$ versus $\left(H_{2}-H_{1}\right)$ for different $T_{\mathrm{cp}}$. The blocking time interval and $T_{\text {cp }}$ are normalized by the data burst transmission time $L$.

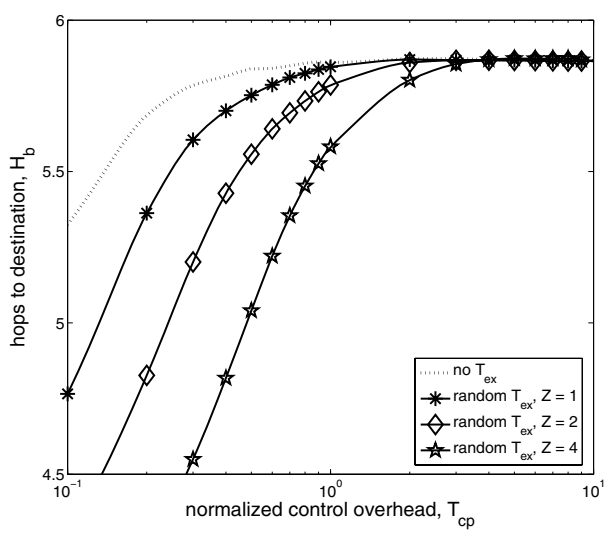

Fig. $4 H_{\mathrm{b}}$ of an eight channel $8 \times 8 \mathrm{MSN}$ with OBS LAUC-VF using different control overhead $T_{\text {cp }}$ when the normalized loading is one. The dotted line represents the simulated $H_{\mathrm{b}}$ of LAUC-VF without random extra offset time $T_{\text {ex }}$. Solid lines with asterisks, diamonds, and pentagrams are the $H_{\mathrm{b}}$ of LAUC-VF with randomized $T_{\mathrm{ex}}$ with $Z=1,2$, and 4 , respectively.

rapidly and becomes zero for $T_{\mathrm{cp}}>L$. For $H_{2}<H_{1}$, the interval increases linearly and becomes $2 L$ when $\left(H_{2}-H_{1}\right) \leq-L / T_{\text {cp. }}$. As $T_{\text {cp }}$ increases, the blocking time interval becomes $2 L$ for all $\left(H_{2}\right.$ $\left.-H_{1}\right)<0$ when $T_{\text {cp }}>L$. Figure 3 shows that a larger $T_{\text {cp }}$ not only strengthens the offset time priority effect, but also increases the probability of a blocked data burst to be blocked by data bursts with larger hop counts.

We define $H_{\mathrm{b}}$ as the average number of hops to the destination of the data burst that blocks a data burst at a node. Figure 4 shows the $H_{\mathrm{b}}$ of an eight channel $8 \times 8 \mathrm{MSN}$ with OBS LAUC-VF using different control overhead $T_{\text {cp }}$ when the normalized loading is one. In Fig. 4, the dotted curve is the simulated $H_{\mathrm{b}}$ of LAUC-VF with different $T_{\mathrm{cp}}$. The value of $H_{\mathrm{b}}$ increases from 5.3 to 5.8 when $T_{\text {cp }}$ increases from 0.1 to 1 . Thus data bursts with larger $H$ will have higher successful probability in channel reservation $\mathrm{w} T_{\text {cp }}$ increases. However, this also increases the bandwidth wastage when these data bursts are blocked. Note also that the OBS LAUC-VF throughput becomes insensitive to the change of $T_{\mathrm{cp}}$ when $T_{\mathrm{cp}}>L$, e.g., the 
curve of $T_{\mathrm{cp}}=10$ in Fig. 1. We have similar observations from Fig. 4. $H_{\mathrm{b}}$ of OBS LAUC-VF will have a value of around 5.8 even if we increase $T_{\text {cp }}$ from 1 to 10 .

\section{A. Methods to improve OBS throughput}

The performance model explains the reasons of OBS to have poor throughput performance as shown in Fig. 1 when $T_{\text {cp }}$ is large. In the following, we briefly discuss different methods to improve the OBS throughput.

1) Constant offset time: Data bursts with different values of $H$ will have the same probability to block each other if the offset time is set to constant. However, FDLs are required at the inputs of the nodes which will increase the complexity of OBS node architecture [2], [3].

2) Dynamic offset time adjustment: In principle, we can dynamically set the initial offset time between data bursts and control packets according to the network traffic condition to reduce the data burst discard rate. Similar to adaptive routing [2], the improvement in performance highly depends on the accuracy of the traffic distribution information. This method is not suitable for systems with highly dynamic traffic and large propagation delay between nodes.

3) Window-based channel reservation: We have investigated a window-based reservation scheme that does not require FDLs [9]. The proposed scheme delays the control packets in a node such that the channel assignment is better than that of the FCFS approach. Since additional delay at a node is equivalent to lengthening $T_{\mathrm{cp}}$, this method is appropriate for the OBS system if the original $T_{\mathrm{cp}}$ is larger than the data burst transmission time.

4) Randomized extra offset time: Throughput improvement has been observed with an extra randomized offset time [10] and this is attributed to the traffic shaping effect of the data bursts at OBS source nodes. However, we find that the randomized extra offset time also significantly weakens the connection between $H$ and the offset time, and hence reduces the blocking. The randomized extra offset time will be discussed in more detail in the next section.

\section{B. Randomized extra offset time}

When a randomized extra offset time is added to Eq. (1), the offset time becomes $T_{\text {off }}=H \times T_{\mathrm{cp}}+T_{\mathrm{sw}}+T_{\mathrm{ex}}$, where $T_{\mathrm{ex}}$ is the random extra offset time. The difference of the two offset times $T_{\mathrm{f} 2}$ and $T_{\mathrm{f} 1}$ is

$T_{\mathrm{f} 2}-T_{\mathrm{f} 1}=\left(H_{2}-H_{1}\right) T_{\mathrm{cp}}+T_{\text {diff }}$

where $T_{\text {diff }}$ is the difference of the two random extra offset times. For $\left(H_{2}-H_{1}\right) T_{\mathrm{cp}}>-T_{\text {diff, }}$ a non-zero time interval is obtained in Eq. (3) only if

$-\left(H_{2}-H_{1}\right) T_{\mathrm{cp}}<T_{\text {diff }}<L-\left(H_{2}-H_{1}\right) T_{\mathrm{cp}}$.

In contrast, Eq. (4) gives a non-zero time interval for any $T_{\text {diff }}<$ $-\left(H_{2}-H_{1}\right) T_{\mathrm{cp}}$. As we assign $T_{\mathrm{ex}}$ at random, $T_{\text {diff }}$ is independent of $\left(H_{2}-H_{1}\right)$, and has the probability density function (pdf) $f_{\text {diff }}(y)=\int_{-\infty}^{\infty} f_{\text {ex }}(y+x) f_{\text {ex }}(x) d x$

where $f_{\mathrm{ex}}(x)$ is the pdf of $T_{\mathrm{ex}}$. For illustration convenience, we simply assume $T_{\mathrm{ex}}$ to be a uniform random variable defined on $(0, Z)$, i.e., $f_{\mathrm{ex}}(x)=1 / Z$ for $0<x<Z$ and zero otherwise. Then $T_{\text {diff }}$ is a random variable defined on $(-Z, Z)$ with the pdf

$f_{\text {diff }}(y)=(Z-|y|) / Z^{2}$ for $-Z<y<Z$,

and zero otherwise. With $\left(H_{2}-H_{1}\right) T_{\mathrm{cp}}=L$, a data burst of $H_{2}$ will not be blocked by any data burst of $H_{1}$ if the offset time is from Eq. (1), i.e., no non-zero time interval will be found from Eqs. (3) and (4). With randomized extra offset time, however, the probability of having a non-zero time interval is 0.5 if the pdf of $T_{\text {diff }}$ is from Eq. (10) and $Z=L$. In Fig. 3, solid lines with asterisks, diamonds, and pentagrams are the $H_{\mathrm{b}}$ of OBS LAUC-VF with randomized extra offset time and $Z=1,2$, and 4, respectively, for the eight channel $8 \times 8 \mathrm{MSN}$ for different $T_{\text {cp. }}$. It shows that $H_{\mathrm{b}}$ can be significantly reduced with large random extra offset times. An average delay of $Z / 2$ however is added to the data bursts.

\section{Performance Evaluation}

Owing to the page length limitation, only the throughput improvement of randomized extra offset time approach is demonstrated in this paper. A detail comparison between different methods listed in Section III-A will be provided in a later paper. In this section, we use simulations to investigate the throughput of OBS LAUC-VF on an $8 \times 8$ Manhattan Street Network (MSN) [7] with different control overhead $T_{\text {cp }}$ and extra random offset time $T_{\text {ex }}$. We focus on the system throughput performance since the average added delay is simply $Z$ / 2 for the data bursts.

In the simulations, data bursts arrive as a Poisson process. When a new data burst arrives at a node, it randomly chooses a destination from the rest of the nodes in the network and uses minimum hop routing to determine the paths. The length of each data burst (transmission time) is one time unit, i.e., $L=1$. To focus on the effect of random extra offset time, no traffic shaping (smoothing the control packet transmissions as in [10]) is applied at the source. After a new data burst is generated at a node, the control packet is sent out immediately to reserve the required wavelength channels on the path. The data burst is then transmitted after the sum of a time period from Eq. (1) (with $T_{\mathrm{sw}}$ $=0$ ) and a random extra offset time $T_{\mathrm{ex}}$. In the simulations, the extra offset time is a random value in $(0, Z)$, where $Z$ is 1,10 , and 100 . If two or more wavelength channels are available, LAUC-VF scheme is used to choose a suitable one. The normalized load offered to a node is the ratio of the average data burst length to the inter-arrival time normalized by the number of channels per link. In the simulations, we assume eight channels per link and all nodes receive the same offered load. The propagation delay of a link is 10 time units. All simulations are 


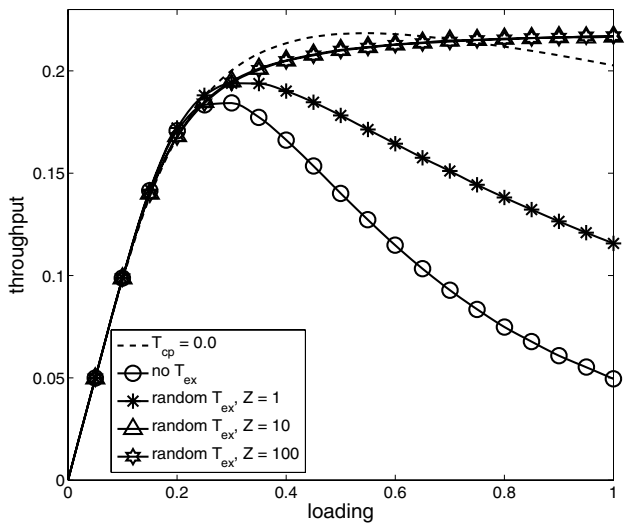

Fig. 5 The throughput of the LAUC-VF with different values of $T_{\mathrm{ex}}$ and $Z$. The control overhead $T_{\mathrm{cp}}=0.1$.

run sufficiently long such that the $95 \%$ confidence intervals are less than $1 \%$ of the average values of the results.

Figures 5 and 6 show the throughput of LAUC-VF with different values of $T_{\mathrm{ex}}$ and $Z$, and the control overhead $T_{\mathrm{cp}}=0.1$ and 1.0 , respectively. In the figures, solid lines with circles, asterisks, triangles, and hexagrams are the throughput of LAUC-VF with no random $T_{\mathrm{ex}}$, and that with random $T_{\mathrm{ex}}$ of $Z=1,10$ and 100, respectively. The dashed line is the throughput of LAUC-VF for $T_{\mathrm{cp}}=0$. From the figures, we observe that the random extra offset time approach can significantly reduce the negative impact of OBS control overhead if the added delay is not a concern. The throughput improvement however diminishes when $Z$ increases. The throughput improves significantly when $Z$ increases from a $T_{\mathrm{cp}}$ to $10 T_{\mathrm{cp}}$, but there is little additional improvement when $Z$ increases from $10 T_{\mathrm{cp}}$ to a larger value.

With a transmission rate of $10 \mathrm{Gps}$ and a data burst size of $100 \mathrm{~K}$ bytes $(L=80 \mu \mathrm{sec})$, the average delays added to the data bursts are 40, 400, and $4000 \mu \sec$ for $Z=1,10$ and 100, respectively. The randomized extra offset time approach is therefore appropriate for OBS networks with millisecond propagation delay between nodes such that the additional delay is negligible.

\section{CONCLUSION}

A large control packet processing time $T_{\text {cp }}$ can severely degrade the throughput performance of optical burst switching (OBS) system. $T_{\text {cp }}$ will become increasingly significant because the data burst transmission time will be shortened with the increase in optical fiber transmission rate. We study the relationship between control packet processing time $T_{\text {cp }}$ and OBS throughput. We show that data bursts with large hop count path are more likely to successfully reserve the output channels at nodes in the early stage of their journey, but they are also likely to be blocked near the destination. Much transmission bandwidth will be wasted and system performance is degraded. Different methods have been discussed to reduce the impact of large $T_{\mathrm{cp}}$. Among the methods, the random extra offset time is discussed in detail because of its simplicity. We show that the

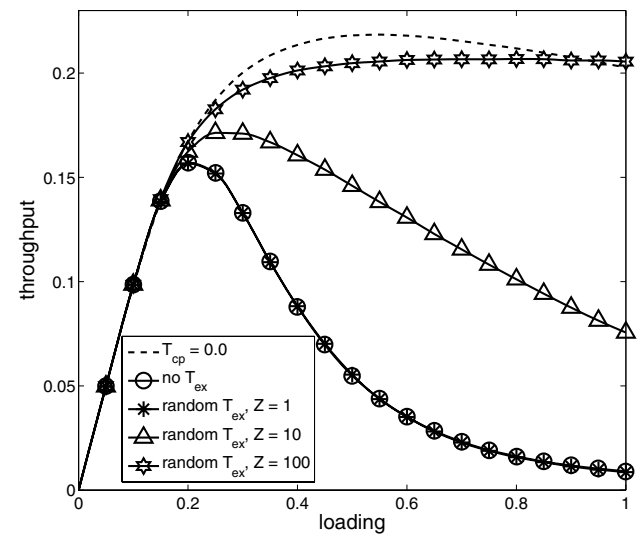

Fig. 6 The throughput of the LAUC-VF with different values of $T_{\mathrm{ex}}$ and $Z$. The control overhead $T_{\mathrm{cp}}=1.0$.

throughput can be significantly improved at the expense of an increase in the data burst delay.

\section{REFERENCES}

[1] T. Houbavlis, et al., "All-optical signal processing and applications within the Esprit project DO_ALL," Journal of Lightwave Technology, Vol. 23, No. 2, pp. 781-801, 2005.

[2] C. Qiao, and M. Yoo, "Optical burst switching (OBS) - a new paradigm for an optical Internet," Journal of High Speed Networks, Vol. 8, pp. 69-84, 1999.

[3] J.S. Tuner, "Terabit burst switching," Journal of High Speed Networks, Vol. 8, pp. 3-16, 1999.

[4] J.Y. Wei, and R.I. McFarland, Jr., "Just-In-Time Signaling for WDM Optical Burst Switching Networks," Journal of Lightwave Technology, Vol. 18, No. 12, pp. 2019-2037, 2000.

[5] Yijun Xiong, M. Vandenhoute, and H.C. Cankaya, "Control architecture in optical burst-switched WDM networks," IEEE Journal on Selected Areas in Communications, Vol. 18, No. 10, pp. 1838-1851, 2000.

[6] C.Y. Li, G.M. Li, P.K.A. Wai, and V.O.K. Li, "Optical burst switching with large switching overhead," IEEE Journal of Lightwave Technology, Vol. 25, No. 2, pp. 451-462, February 2007.

[7] N.F. Maxemchuk, "Routing in Manhattan Street network," IEEE Transactions on Communications, Vol. 35, No. 5, pp. 503-512, 1987.

[8] B.C. Kim, Y.Z. Cho, and D. Montgomery, "An efficient optical burst switching technique for multi-hop networks," IEICE Transactions on Communications, Vol. E87-B, No. 6, pp. 1737-1740, 2004.

[9] C.Y. Li, G.M. Li, P.K.A. Wai, and V.O.K. Li, "Novel resource reservation schemes for optical burst switching," Proceedings of IEEE International Conference on communications (ICC 2005), Vol. 3, pp. 1651-1655, Seoul, Korea, May 16-20, 2005.

[10] S. Verma, H. Chaskar, and R. Ravikanth, "Optical burst switching: a viable solution for terabit IP backbone," IEEE Network, Vol. 14, No. 6, pp. $48-53,2000$. 